\title{
Study on the Deformation and the Test of the Electrostatic Driven MEMS Flexible-Beam
}

\author{
Qing-Ling LIU \\ LangFang Teachers University, LangFang, Hebei, China 065000 \\ lql1224@163.coml
}

Keywords: Electrostatic Driven, Flexible Beam, Deformation, Pull-in, Test.

\begin{abstract}
Based on the theory about the electrostatic, the non-uniformly distributed load and the coupling between the electrostatic force and the deformation of the flexible beam are considered, the deformation analysis of the electrostatic driven flexible beam is done, the mathematic model about the deformation of the flexible beam under different driven voltage is established and the equation for calculating the pull-in voltage is given. The flexible beam is designed and machined, its deformation are analyzed with the above method and model. The test system is built, the deformation of the flexible beam under different driven voltage and the pull-in voltage are tested, which contrasted with the theoretical results. The validity of the mathematic model above is proved.
\end{abstract}

\section{Introduction}

With the development of MEMS and the micro-nano test technology, the driving methods are developed. The electrostatic driven is widely used in MEMS compliant structures because of its large driven work range, simple loading mode and it is easier integrated with microelectronics.

Compliant structure is the most important component in the MEMS mechanism, the flexible beam is one of the most commonly used structure form in the micro-actuator and micro-sensor. The electrostatic driven exists the coupling between the deformation of the flexible beam and the electricity, the deformation is nonlinear. When the driven voltage exceeds a certain limit, the pull-in will produce between the flexible beam and the fixed electrode which is one of the important characteristic in the electrostatic driven. The study on the electrostatic driven, the electricity was discreted and was considered as point load, in fact, it is non-uniform distribution load, so in this paper a new method is proposed and the mathematical model is established used for calculating the deformation of the electrostatic driven flexible beam, the equation used for calculating the pull-in voltage is established, which can be used for providing the reasonable driven voltage.

\section{The Deformation of the Electrostatic Driven MEMS Flexible Beam}

The capacitor structure are formed with the electrostatic driven flexible beam and the fixed electrode, which is shown in Fig 1.

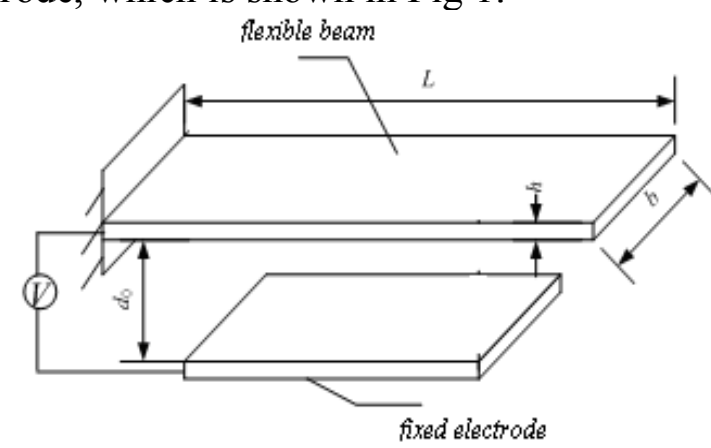

Fig.1 The Structure Sketch of the Electrostatic Driven Electrostatic Driven MEMS Flexible Beam

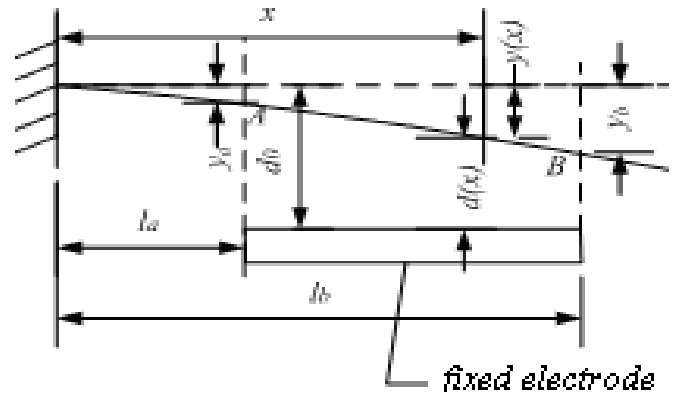

Fig. 2 The Sketch of the Deformation of the MEMS Flexible Beam 


\section{The Mathematical Model about the Deformation of the Electrostatic Driven Flexible Beam}

Based on the capacitor theory, the static electricity between the infinite plate capacitor is determined as:

$$
F e=\frac{\varepsilon a b U^{2}}{2 d^{2}}
$$

Where, $\varepsilon$ is the dielectric constant; a,b are the length and width of the capacitor plate; $\mathrm{U}$ is the voltage between the capacitor plate; $d$ is the distance between the capacitor plate;

The voltage is applied to the capacitor shown in Fig1, the flexible beam is deformed, the static electricity is non-uniform distribution load. The center line of the flexible beam is used for denoting its deformation position which shown in the Fig2, the dotted line and the solid line denote respectively the position of the flexible beam before and after deformation.

Take a point on the flexible beam away from the fixed end of the flexible beam $\mathrm{x}$, the deformation of the point and the distance between the point and the fixed electrode are denoted respectively as $\mathrm{y}(\mathrm{x})$ and $\mathrm{d}(\mathrm{x})$ which shown in Fig2, so the static electricity of the point can be determined as:

$$
F e(x)=\frac{\varepsilon b U^{2}}{2[d(x)]^{2}}=\frac{\varepsilon b U^{2}}{2\left[d_{0}-y(x)\right]^{2}}
$$

Where, ${ }^{d_{0}}$ is the initial distance between the flexible beam and the fixed electrode;

In Fig2, the deformation of the point $\mathrm{A}$ and $\mathrm{B}$ on the beam is denoted as $y_{a}, y_{b}$, so the deformation of the point away from the fixed end $\mathrm{x}$ is determined as:

$$
y(x)=y_{a}+\dot{y}_{a}\left(l_{a}-x\right)
$$

Where, $\dot{y}_{a}$ is the slope of the point A after deformation;

Choosing tiny parts $\mathrm{dx}$ on the beam away from the fixed end of the beam $\mathrm{x}$, so the static electricity can be determined as:

$$
d F e=\frac{\varepsilon b U^{2}}{2\left(d_{0}-y(x)\right)^{2}} d x=\frac{\varepsilon b U^{2}}{2\left[d_{0}-\left(y_{a}+\dot{y}_{a}\left(l_{a}-x\right)\right)\right]^{2}} d x
$$

Integrating to equation (4), so the static electricity of the flexible beam can be determined as:

$$
F e=\int_{l_{a}}^{l_{b}} \frac{\varepsilon b U^{2}}{2\left[d_{0}-\left(y_{a}+\dot{y}_{a}\left(l_{a}-x\right)\right)\right]^{2}} d x=\frac{\varepsilon b U^{2}\left(l_{b}-l_{a}\right)}{2\left(d_{0}-y_{a}\right)\left[d_{0}-\left(y_{a}+\dot{y}_{a}\left(l_{b}-l_{a}\right)\right)\right]}
$$

Because of the scale effect, the deformation and the static electricity of the electrostatic driven flexible beam are all very little. The slope of the deformation of the flexible beam is approximately represented with the tangent of the angle deformation, so the static electricity can be determined as:

$$
F_{e}==\frac{\varepsilon b U^{2}}{2} \frac{1}{\operatorname{tg} \theta}\left(\frac{1}{d_{0}-l_{b} \operatorname{tg} \theta}-\frac{1}{d_{0}-l_{a} \operatorname{tg} \theta}\right)
$$

Where, $l_{a}, l_{b}$ are the distance between the left and right end of the fixed electrode and the fixed end of the flexible beam;

The static electricity torque of the flexible beam can be determined as: 


$$
M_{e}=\int_{l_{a}}^{l_{b}} x F e(x) d x=\frac{\varepsilon b U^{2}}{2} \frac{1}{\operatorname{tg}^{2} \theta}\left[\ln \left(\frac{d_{0}-l_{b} \operatorname{tg} \theta}{d_{0}-l_{a} \operatorname{tg} \theta}\right)+d_{0}\left(\frac{1}{d_{0}-l_{b} \operatorname{tg} \theta}-\frac{1}{d_{0}-l_{a} \operatorname{tg} \theta}\right)\right]
$$

Suppose the equivalent stiffness of the flexible beam is $\mathrm{k}$ and the angle deformation of the flexible beam is $\theta$ under a certain voltage, based on the torque balance, the equation can be determined as:

$$
k \theta=M e
$$

With the equation (7) and (8), the equation can be determined as:

$$
k \theta=\frac{\varepsilon b U^{2}}{2} \frac{1}{\operatorname{tg}^{2} \theta}\left(\ln \left(\frac{d_{0}-L_{b} \operatorname{tg} \theta}{d_{0}-L_{a} \operatorname{tg} \theta}\right)+d_{0}\left(\frac{1}{d_{0}-L_{b} \operatorname{tg} \theta}-\frac{1}{d_{0}-L_{a} \operatorname{tg} \theta}\right)\right)
$$

With the equation (9), the angle deformation of the flexible beam under a certain voltage can be determined as:

$$
\frac{\varepsilon b U^{2}}{2 k}=\frac{\theta \times \operatorname{tg}^{2} \theta}{\ln \left(\frac{d_{0}-L_{b} \operatorname{tg} \theta}{d_{0}-L_{a} \operatorname{tg} \theta}\right)+d_{0}\left(\frac{1}{d_{0}-L_{b} \operatorname{tg} \theta}-\frac{1}{d_{0}-L_{a} \operatorname{tg} \theta}\right)}
$$

With equation (6) and (7) and the angle deformation, the static electricity and static electricity torque under a certain voltage can be calculated.

\section{The Pull-in Voltage of the Electrostatic Driven Flexible Beam}

The deformation will increase with the increase of the driven voltage which will lead to the static electricity increasing more quickly, so the pull-in will happen between the flexible beam and the fixed electrode. Supposed as follow with equation (10):

$$
f(\theta)=\frac{\theta \times \operatorname{tg}^{2} \theta}{\ln \left(\frac{d_{0}-L_{b} \operatorname{tg} \theta}{d_{0}-L_{a} \operatorname{tg} \theta}\right)+d_{0}\left(\frac{1}{d_{0}-L_{b} \operatorname{tg} \theta}-\frac{1}{d_{0}-L_{a} \operatorname{tg} \theta}\right)}
$$

Differentiated to Equation (10), the equation can be determined as:

$$
f^{\prime}(\theta) d \theta=\frac{\varepsilon b U}{k} d U, \quad \frac{d U}{d \theta}=\frac{f^{\prime}(\theta)}{\frac{\varepsilon b U}{k}}
$$

Suppose $\frac{d U}{d \theta}=0$, based on $f^{\prime}(\theta)=0$, the critical angle deformation $\theta_{c}$ in pull-in can be determined.

With the $\theta_{c}$ and equation (10), the pull-in voltage $U_{c}$ can be determined as:

$$
U_{c}=\sqrt{\frac{\theta_{c} \times t^{2} \theta_{c}}{\ln \left(\frac{d_{0}-L_{b} \operatorname{tg} \theta_{c}}{d_{0}-L_{a} \operatorname{tg} \theta_{c}}\right)+d_{0}\left(\frac{1}{d_{0}-L_{b} \operatorname{tg} \theta_{c}}-\frac{1}{d_{0}-L_{a} \operatorname{tg} \theta_{c}}\right)}}
$$

With the pull-in voltage and the breakdown voltage, the electrostatic driven voltage range can be determined as: $0 \leq U \leq \min \left(U_{c}, U_{\text {breakdown }}\right)$ 


\section{The Deformation Analysis of the Electrostatic Driven MEMS Flexible Beam}

\section{The Example of the MEMS Flexible Beam}

MEMS flexible beam is designed and machined whose material is monocrystalline silicon, Young's modulus E=169GPa. The flexible beam and its size are shown in Fig3, the width of the beam is $75 \mu \mathrm{m}$.

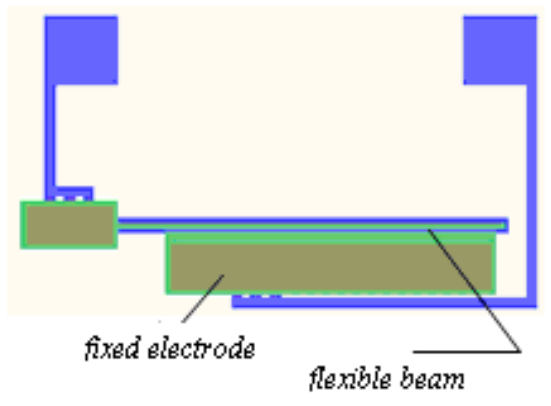

(a) The design chart of the flexible beam

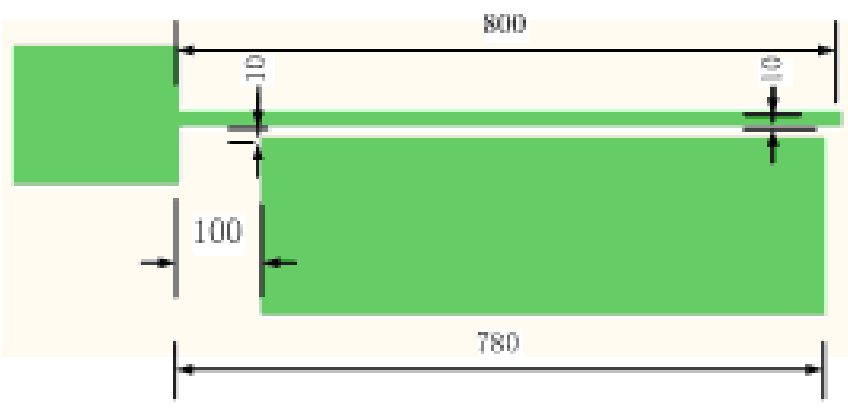

(b)The size of the flexible beam(unit: $\mu \mathrm{m}$ )

Fig. 3 The Design Chart and Its Size of the Flexible Beam

\section{The Theoretical Deformation Analysis of the Electrostatic Driven MEMS Flexible Beam}

The angle deformation is calculated under different voltage with equation (10) and the size of the flexible beam shown in Fig3, so the deformation of point B shown in Fig2 is calculated. The results are listed in Table1. The pull-in voltage can be calculated with equation (11): $\mathrm{Uc}=112.8 \mathrm{~V}$.

Tab.1 The Deformation Theoretical Results of Point B under Different Voltage

\begin{tabular}{cccccccccccccc}
\hline Voltage $(\mathrm{V})$ & 50 & 55 & 60 & 65 & 70 & 75 & 80 & 85 & 90 & 95 & 100 & 105 & 110 \\
\hline $\begin{array}{c}\text { Derformation } \\
(\mu \mathrm{m})\end{array}$ & 0.417 & 0.512 & 0.625 & 0.731 & 0.864 & 0.997 & 1.196 & 1.395 & 1.594 & 1.86 & 2.247 & 2.591 & 3.122 \\
\hline
\end{tabular}

\section{The Deformation Test of the Electrostatic Driven MEMS Flexible Beam}

\section{The Test System}

The electrostatic driven voltage is supplied by the DC regulated power is used. Considering the demands about MEMS compliant structure test and voltage supply, the test circuit is designed which shown in Fig4. The test system is built and the local test system are shown in Fig5.

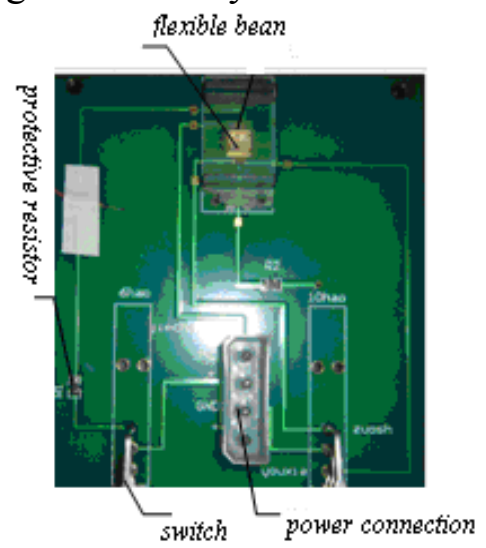

Fig. 4 The Test Circuit

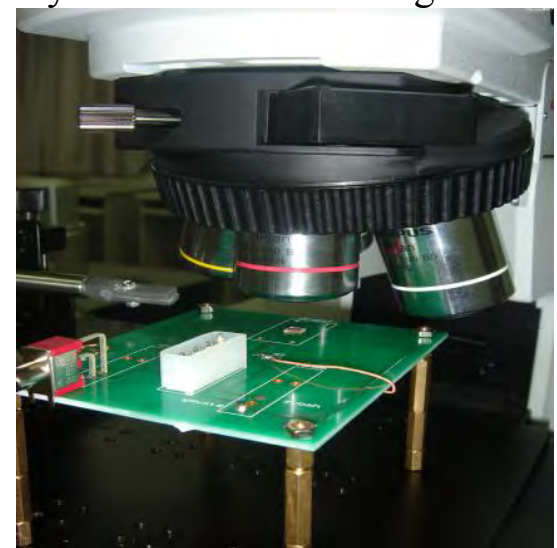

Fig. 5 The Local Test System of Electrostatic Driven 


\section{The Driven Voltage Range}

The theoretical pull-in voltage is calculated by the equation (11): $\mathrm{Uc}=112.8 \mathrm{~V}$. Considering the breakdown voltage of the plate capacitor and Paschen curve, the breakdown voltage is about $330 \mathrm{~V}$. So the driven voltage range is about: $0-110 \mathrm{~V}$.

\section{The Deformation Test of the Flexible Beam}

The deformation of the point $\mathrm{B}$ (Fig2) on the beam is tested. The distance between point $\mathrm{B}$ and the fixed electrode is tested under different voltage, the deformation is the difference between the distance tested above and the initial distance $\mathrm{d} 0$.The driven voltage range is $0-110 \mathrm{~V}$. The voltage is increased from $50 \mathrm{~V}$ to $110 \mathrm{~V}$ gradually, the interval is $5 \mathrm{~V}$. The deformation of the point $\mathrm{B}$ is recorded under different voltage. The distance between the point $\mathrm{B}$ and the fixed electrode under $90 \mathrm{~V}$ is shown in Fig6. The average values of the ten test results are shown in Table2.
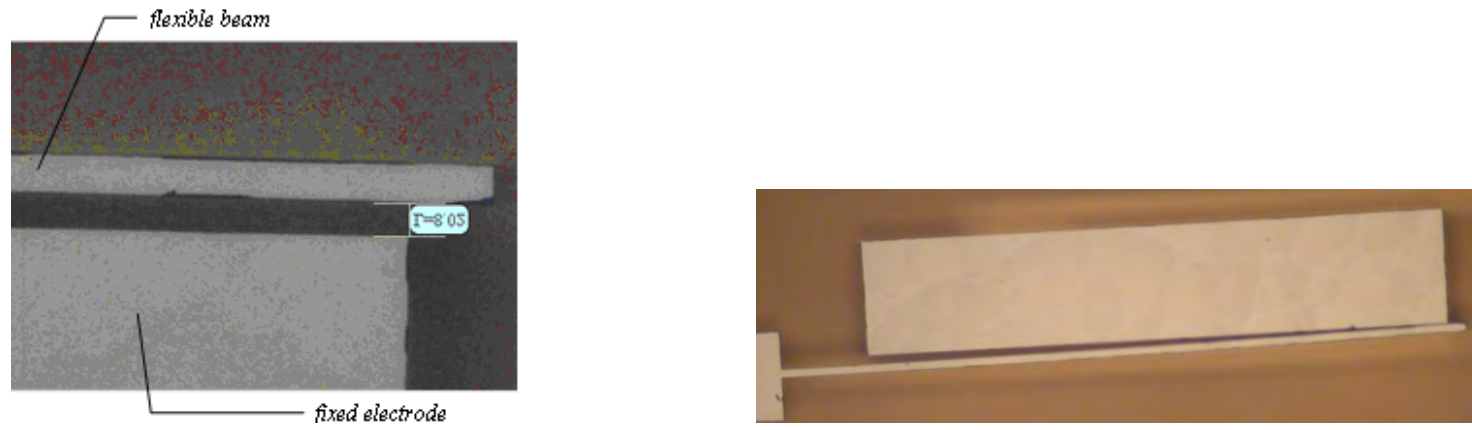

Fig. 6 The Test Distance under 90V (local enlargement) Fig. 7 The Pull-in of the Electrostatic Driven

Tab. 2 the Deformation Test Results of the Point B under Different Voltage

\begin{tabular}{cccccccccccccc}
\hline Voltage $(\mathrm{V})$ & 50 & 55 & 60 & 65 & 70 & 75 & 80 & 85 & 90 & 95 & 100 & 105 & 110 \\
\hline $\begin{array}{c}\text { Distance } \\
(\mu \mathrm{m})\end{array}$ & 9.26 & 9.12 & 8.99 & 8.86 & 8.72 & 8.59 & 8.35 & 8.18 & 8.05 & 7.64 & 7.39 & 7.09 & 6.50 \\
\hline $\begin{array}{c}\text { Deformation } \\
(\mu \mathrm{m})\end{array}$ & 0.40 & 0.54 & 0.67 & 0.80 & 0.94 & 1.07 & 1.31 & 1.48 & 1.61 & 2.02 & 2.27 & 2.57 & 3.16 \\
\hline
\end{tabular}

When the driven voltage is increased to $110 \mathrm{~V}$ and is increased continuously to $114 \mathrm{~V}$, the pull-in between the flexible beam and fixed electrode is happened and is shown in Fig7. The theoretical result is $112.8 \mathrm{~V}$ and its error is about $1.05 \%$.

\section{The Result Analysis}

Based on the results of the table1 and table2, the theoretical result is similar to the experimental test results. The most important error reason is the change of Young's modulus because of micro-machining, the others are the machining error, observation error and reading error.

\section{Conclusion}

1) The deformation characteristic study is done about the electrostatic driven MEMS flexible beam, the distributed load and nonlinear of the static electricity are considered, the mathematical model is established for calculating the deformation of the flexible beam; The pull-in is the important characteristic of the electrostatic driven, the equation about calculating the pull-in voltage is established; The theoretical deformation is calculated with the method above;

2)The MEMS flexible beam is designed and machined, the test system is built, the test circuit is designed. The driven voltage range is decided based on the pull-in voltage and breakdown voltage, the deformation of the flexible beam under different voltages is tested; 
3) The comparative analysis between the theoretical results and the test results is done, the two results are consistent, so the analysis method and mathematical model about deformation of the flexible beam is correct. The error reason is analyzed.

\section{Acknowledgment}

This paper is supported by Dr. fund of LangFang Teachers University (No.LSBS201306).

\section{References}

[1]FANG Dong-ming, FU Shi , ZHOU Yong, etal. A Tunable Micromechanical Capacitor Driven by Electrostatic Force[J]. Journal of emiconductors2007, 28 (9):1454-1458.

[2]JIA Cui-ping, ZHU Hua-feng. Design of Electrostatic Actuator of Optical Switch and Analysis of Actuating Voltage[J]. Journal of Semiconductor Technology. 2010, 35(5):440-442.

[3]Liu YaoBo, Yuan Weizheng, Qiao DaYong. Design and Modesof a Novel Two-Dimensional Microscanner with Electrostatic Actuatioin [J]. ACTA OPTICA SINICA. 2013, 33(6):1-6.

[4]Shang HuiLin, Wen YongPeng. Pull-in stability of electrostatically actuated MEMS resonant sensor and its control[J]. JOURNAL OF VIBRATION AND SHOCK. 2013, 32(15):8-13.

[5]Xin Chao, Li LiWei. Elastic Deformation Analysis of Electrostatic Driving Structure in Different Voltage [J]. Journal of Shanghai University of Electric Power, 2013, 29(4):330-332.

[6]Min Liu, Kurt Maute, Dan M. Frangopol. Multi-objective design optimization of electrostatically actuated Micro-beam resonators with and without parameter uncertainty[J]. Reliability Engineering and System Safety. 2007, 92:1333-1343.

[7]R.C. Batra, M. Porfiri, D. Spinell o. Vibrations of narrow micro-beams pre-deformed by an electric field[J] .Sound and Vibration. 2008, 309:600-612.

[8]Javier Malo, Jose Ignacio Izpura. Simultaneous magnetic and electrostatic driving of micro-cantilevers[J]. Sensors and Actuators A. 2007, 136: 347-357.

[9]L I Yong, L I Yu-he, L I Qing-xiang. Computation of electrostatic forces with edge effects for non-parallel comb-actuators[J]. Journal of TSINGHUA University (Science and Technology), 2003, 43(8):1024-1026.

[10]WU Chong-ruo, FAN Yu-wei. A Study on Gas dis-change Properties in Micro-gaps. Journal of Electron Devices. 1993, 16(2):54-59.

[11]HOU Qing-run, MAO Wei-hong, CHEN Yi-bao.Glow discharge and Paschen law[J]. Journal of Physics Experimentation. 2004, 24(1):4-6. 